Journal of trends in Computer Science and Smart technology (TCSST) (2020)

Vol.02/ No. 01

Pages: 26- 36

https://www.irojournals.com/tcsst/

DOI: https://doi.org/10.36548/jtcsst.2020.1.003

\title{
Resource Intensification for Mobile Devices Using the Approximate Computing Entities
}

\author{
Dr. V Suma, \\ Professor, Department of Information Science \& Engineering \\ Dayananda Sagar College of Engineering \\ Shavige Malleshwara Hills, Kumarswamy Layout \\ Banglore, India-78 \\ Email-ID suma-ise@ dayanandasagar.edu
}

\begin{abstract}
The mobile devices are termed to highly potential due to their capability of rendering services without being plugged to the electric grid. These device are becoming highly prominent due to their constant progress in computing as well as storing capacities and as they are very much closer to the users. Despites its advantages it still faces many problems due to the load balancing and energy consumption due to its limited battery limited and storage availability as some applications or the video downloading requires high storage facilities consuming majority of the energy in turn reducing the performance of the mobile devices. So as to improve the performance and the capability of the mobile devices the mobile cloud computing that integrates the mobile devices with the cloud paradigm has emerged as a promising paradigm. This enables the augmentation of the local resources for the mobile devices to enhance its capabilities in order to improve its functioning. This is basically done by proper offloading and resource allocation. The proposed method in the paper utilizes the optimal offloading strategy (Single and double strand offloading) and follows an Ant colony optimization based resource allocation for improving the functioning the mobile devices in terms of energy consumption and storage.
\end{abstract}

Keywords: Mobile Devices. Cloud Computing, Mobile Cloud Computing, Optimal Offloading, Resource Allocation.

\section{Introduction}

Mobile devices have been commonly used in the real-life, with the rapid growth of electronics technology. There are some drawbacks, however, such as low battery and storage space, limited processing power to restrict their uses. Several capabilities to overcome such bottlenecks have been suggested. One of them is "mobile cloud computing (MCC), the combination of mobile and cloud, which allows mobile devices to

ISSN: 2582-4104 
Journal of trends in Computer Science and Smart technology (TCSST) (2020)

Vol.02/ No. 01

Pages: 26- 36

https://www.irojournals.com/tcsst/

DOI: https://doi.org/10.36548/jtcsst.2020.1.003

collect heavy computing results in a lower configuration". The MCC is termed as the very important instance and one the key innovations in the cloud computing.

Mobile apps leverage external cloud services to increase their computing competencies, for instance space for storing, and utilize the local services. "Cloud computing provides efficient and flexible tools for lowcost usage when required, mobile apps use the distributed computing model to achieve improved user experience and high performance through the use of cloud services anytime and anywhere. This is an advancement of various other technologies, such as grid computing, parallel computing and distributed computing. Considering the benefits of MCC, mobile users can connect to the cloud server remotely and achieve maximum computing power as opposed to doing it close by". To increase and maximize the computing abilities in the mobile devices the offloading strategy is plays a vital role in the MCC. This strategy involves segregating and examining all the applications of mobile. "Then, this application's most resource-intensive components are detected and remotely offloaded to the chosen, efficient cloud server. This will then perform the required calculation and return the results to the mobile end-client".

One such high popular example is the mobile health care applications. These application produce enormous amount of data that utilizes majority of the mobile resources and deteriorates the performance of the device even if is equipped with the high computational capabilities and storage. So the enormous data generated are offloaded to the cloud, processed and sends back the mobile users holding the application. "The transfer of heavy computation from mobile devices via communication networks to remote cloud servers could be seen as a straightforward process. Nevertheless, the diversity in MCC affects the consumption of mobile cloud services in real time as the cloud services selection process depends on the multiple services available in the MCC paradigm which belong to heterogeneous environments".

So these issues makes necessary the optimization in the energy consumption in selecting scheme for computing. Before the offload calculation is carried out, there are two critical stages to process: code segregation and portion migration. "Many experiments have been carried out to settle on the optimal offloading strategy, respectively by linear complexity algorithm and heuristic algorithm". MCC Paradigm Environments. Though several researches have been performed and the algorithms with the linear complexity and the heuristics have been formulated to identify the optimal strategies the complexities still exists in sorting out the offloading schemes for the general applications this makes it necessary to transform the general applications into chain of applications. The paper puts forth the optimal offloading scheme combining the single and the multi-stranded computing followed by resource allocation employing the Ant colony optimization-ACO.

ISSN: 2582-4104 
Journal of trends in Computer Science and Smart technology (TCSST) (2020)

Vol.02/ No. 01

Pages: 26- 36

https://www.irojournals.com/tcsst/

DOI: https://doi.org/10.36548/jtcsst.2020.1.003

The paper is organized with the types of offloading strategies and their applications in the section 2 that presents the related works, section 3 presenting the proposed work, section 4 providing the performance analysis and section 5 with the conclusion followed by the references.

\section{Related works}

Based on the type of information's migrated the offloading scheme is categorized into two types as local and complete. The local offloading scheme just transmits the data that has the high energy consumption to the cloud server reducing the amount of data transmission. Complete offloading transmits entire program to the cloud unlike local and consumes majority of energy while transmitting. The author Tao et al [1] tries to balance the energy consumption and the latency by putting forth two offloading schemes that are optimal, the author evaluated the proposed model utilizing the numerical experiments. Hyytiä et al [2] the author discloses a near-optimal offloading policies to minimize the cost, the expected latencies and the power consumption.

Smys, S et al [3] puts forth the data traffic model that delivers security, longevity, convenience and safety, estimating the throughput, PDR and the latencies. Deng et al [4] puts forth a robust offloading strategy based on the genetic algorithm to handle the specific demands of the offloading and further optimizes the time expected for execution as well as energy usage. Bhalaji, N. et al [5] performed the "Delay Diminished Efficient Task Scheduling and Allocation for Heterogeneous Cloud Environment"

Ma et al [6] the paper analyses the offloading issues in the MCC-cloudlets by examining the offloading schemes of the multiple clients engaging the game theory. Karunakaran et al [7] has disclosed "A Stochastic Development of Cloud Computing Based Task Scheduling Algorithm" Barbarossa et al [8] and [9] presents the offloading optimizing the energy usage and latency and cost and further proposes a cross layer optimization for transferring the load of the mobile devices to cloud.

Bashar, A et al [10] presents the "Secure and Cost Efficient Implementation of the Mobile Computing Using Offloading Technique" Gao et al [11] organizes an energy efficient work flow by framing an strategy to offload from the mobile to cloud optimizing the energy. Khan, et al [12] elaborates the "survey of computation offloading strategies for performance improvement of applications running on mobile devices". Kamoun, et al [13] proposes the "Joint resource allocation and offloading strategies in cloud enabled cellular networks."

ISSN: 2582-4104 
Journal of trends in Computer Science and Smart technology (TCSST) (2020)

Vol.02/ No. 01

Pages: 26- 36

https://www.irojournals.com/tcsst/

DOI: https://doi.org/10.36548/jtcsst.2020.1.003

Ge, et al [14] has conducted the allocation of resources for the MCC by utilizing the game theory approach. Shi et al [15] has devised a strategy to offload based on the mobility of the mobile devices for the MCC that is distributed. Wu et al [16] has conducted the "Analysis of the energy-response time tradeoff for mobile cloud offloading using combined metrics." Xia, et al [17] has devised a "Single and DoubleThreaded Offloading Strategies and Computational Analysis in Mobile Cloud Computing." Feng et al [18] has formulated a "Autonomous vehicular edge computing framework with ACO-based scheduling"

The proposed method in the paper forms a offloading system as in [4], further utilizes the offloading scheme put forth on the [17] and allocates the resources based on the ACO in [18] The following section shows the phases of the proposed scheming for discharging tasks of mobile to cloud

\section{Proposed system}

The proposed off loader is framed into a system [4] with four major phases as shown in the figure.1, with the initial phase doing the process of observing, and performing a real time data gathering from the mobile networks as well as devices and the cloud servers, followed by the organizer that conceives a "specific double threaded calculation scheme for the offloading" as in [17]. Further the resources allocation based on the foraging behavior of the Ant [18] is put forth the third stage and finally executes the requests of the mobile according to the decisions done in the organizer.

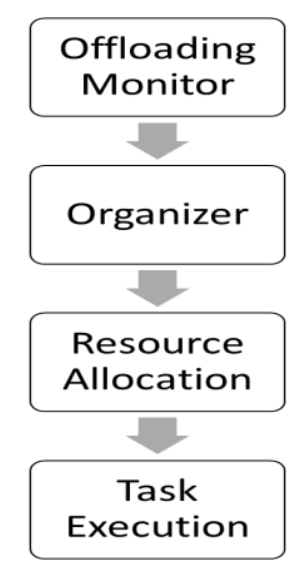

Figure.1 off -Loader System

ISSN: 2582-4104 
Journal of trends in Computer Science and Smart technology (TCSST) (2020)

Vol.02/ No. 01

Pages: 26- 36

https://www.irojournals.com/tcsst/

DOI: https://doi.org/10.36548/jtcsst.2020.1.003

The "offloading monitor, keeps note on the activities of the mobile devices and collects the particulars from the mobile devices as well as network and from the cloud server" the simple application and the general application in [1] is considered and are combined to form a "double stranded estimation of the offloading scheme" this is done in the organizer phase of the off-loader system. "The single chained or the simple application allows at most only one migration from the device to the cloud". Whereas the general applications segregates the load into two sub-division $\left\{\right.$ node $_{0}$ and node $\left.e_{n+1}\right\}$, represented as the section one with the lower loads and section 2 larger loads and the average count of the transferred data across the two sections must be maintained low. More over the node contraction is done by replacing the two nodes $n_{1}$ and the $n_{2}$ with the $\dddot{n}_{i}$, if the above mentioned two nodes union are adjacent to the $\dddot{n}_{i}$, the nodes here are considered as the virtual components, the virtual components are combined to bring down the total cost.

The proposed frame work takes into consideration both the single and the general application and forms the combined strategy for offloading, the figure. 2 below shows the combined simple application and the general application to form the double stranded estimation method for off-loading.

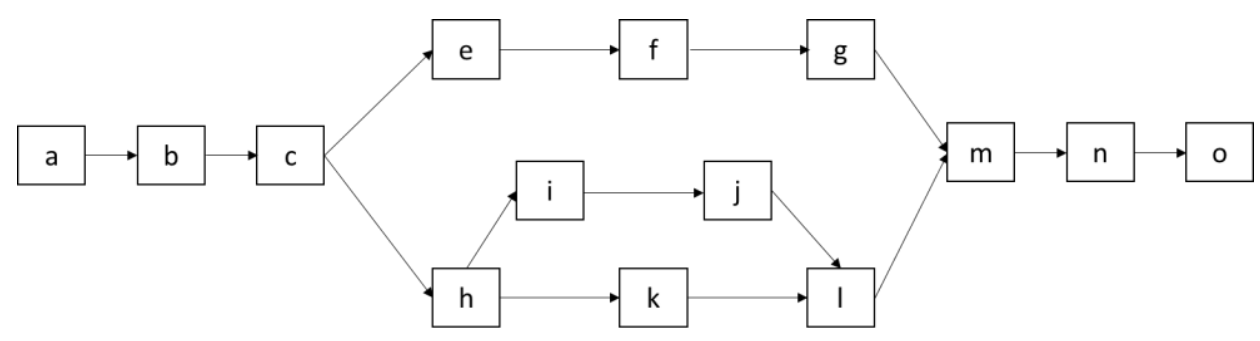

Figure.2 Double Stranded Estimation Scheme Observed From [17] and Modified

The scheme to be off-loaded is depicted as $\left\{O l_{S c}=\left(O l_{S c}(a), \ldots \ldots .(x),(x+1)\right\}\right.$ when the decision is made to off load the data $(\mathrm{x})$ the ' $\mathrm{X}$ ' is set either as 1 or 0 and assumed as $\mathrm{X}=0=\mathrm{X}+1=0$. Initially using the simple application the total count of segments are minimized to reduce the energy usage and uses the genera application to minimize the strand frequency to reduce the energy usage of the mobile devices reducing the estimation frequency. So the objective functioning to devise the offloading for the mobile device is estimated using the overall energy consumed $\left(E_{c}\right)$, the total execution time $\left(E_{T}\right)$ and the weight co-efficient defined using the status of the mobile $\left(W_{s}\right)$, as shown in equation 1

ISSN: 2582-4104 
Journal of trends in Computer Science and Smart technology (TCSST) (2020)

Vol.02/ No. 01

Pages: 26- 36

https://www.irojournals.com/tcsst/

DOI: https://doi.org/10.36548/jtcsst.2020.1.003

$$
F\left(O L_{M}\right)=E_{c}\left(1-W_{s}\right)+E_{T}\left(W_{s}\right)
$$

Once the offloading is scheme is devised the resource allocation is done applying the foraging behavior of the ant as in the [18] the resource allocation is also an multi objective optimization as the it is necessary to identify the resources that are compatible to perform the tasks, so as a first step the particulars of the tasks ( task size $\left(\operatorname{task}_{\text {size }}\right)$, computational requirements $\left(\mathrm{Com}_{\text {req }}\right)$ that involves the energy requirement, memory requirements and the processing capacity needed, and the dead line $\left(D_{L}\right)$ of the task) and the particulars of the resources ( memory capacity $\left(M_{c a p}\right)$, and the processing speed $\left(\right.$ Pro $\left._{\text {Speed }}\right)$ ) and the resource allocation is performed using the ACO. The algorithm below in figure. 3 is the ACO based resource allocation.

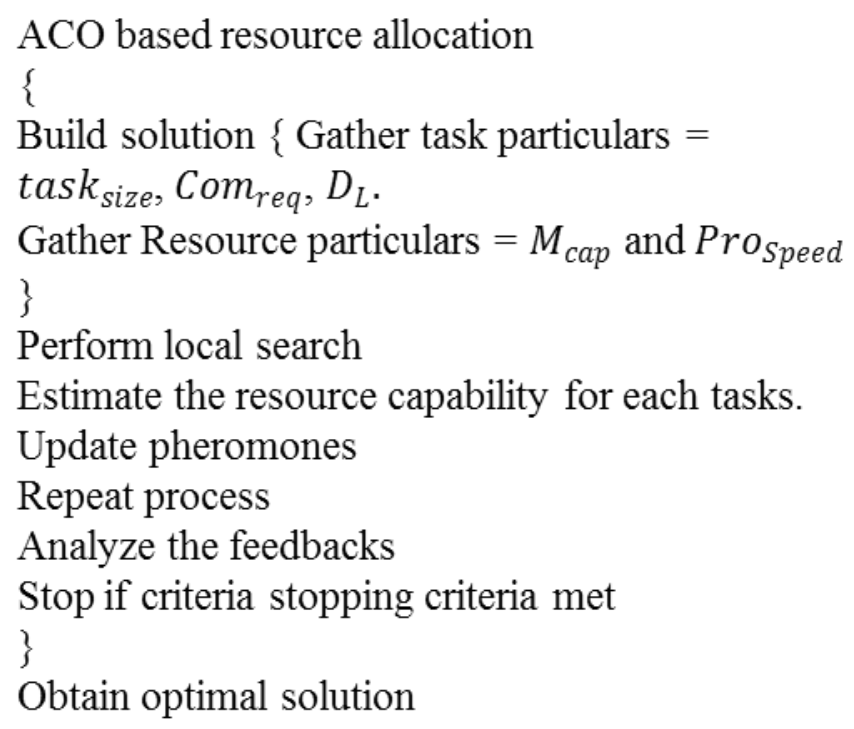

Figure.3 ACO Based Resource Allocation

Followed by the resource allocation the task are executed and sent back to the mobile devices.

ISSN: 2582-4104 
Journal of trends in Computer Science and Smart technology (TCSST) (2020)

Vol.02/ No. 01

Pages: 26- 36

https://www.irojournals.com/tcsst/

DOI: https://doi.org/10.36548/jtcsst.2020.1.003

\section{Results Evaluation}

The proposed Off-loading scheme is simulated in cloudsim and evaluated with varying number of tasks and resources for a duration of 10000 seconds, on the basis of energy consumption, the time taken for execution and the cost incurred. The evaluation results of the proposed frame work is compared with the evaluation results of the ETSA put forth in [5] and SDTS in [7] the table.1below shows the parameter used in the simulation.

\begin{tabular}{|c|c|}
\hline Parameter & Value \\
\hline Processing Time & 10000 \\
\hline Available Servers & 20 \\
\hline Tasks & 100 \\
\hline Resources & 20 \\
\hline Path loss & $128+38 \log _{10}$ \\
\hline P2P link loss & $148+40 \log _{10}$ \\
\hline
\end{tabular}

Table.1 Simulation Parameter

The figure. 4 is the energy consumed by the proposed method and its comparison with the existing method is shown in the ETSA and SDTS, the proposed offloading shows considerable reduction in the energy consumption than the prevailing methods.

ISSN: 2582-4104 
Journal of trends in Computer Science and Smart technology (TCSST) (2020)

Vol.02/ No. 01

Pages: 26- 36

https://www.irojournals.com/tcsst/

DOI: https://doi.org/10.36548/jtcsst.2020.1.003

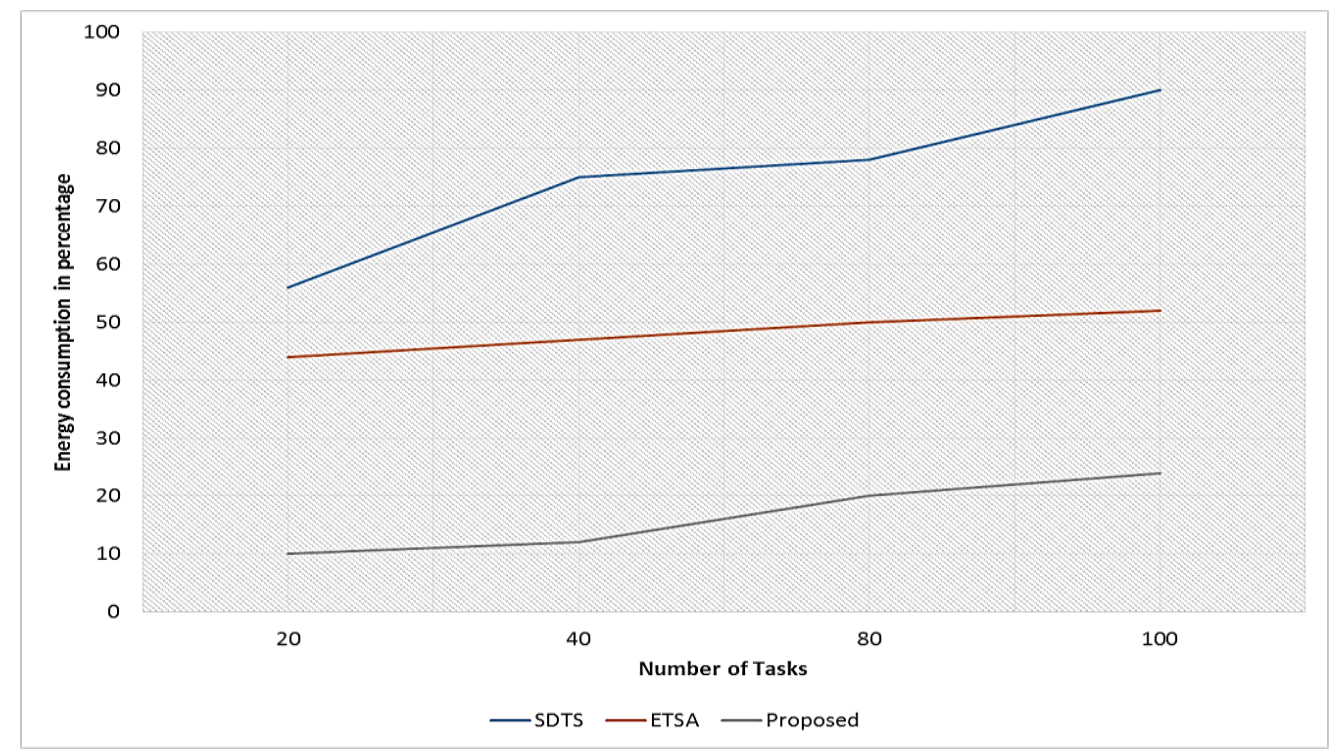

Figure.4 Energy Consumed

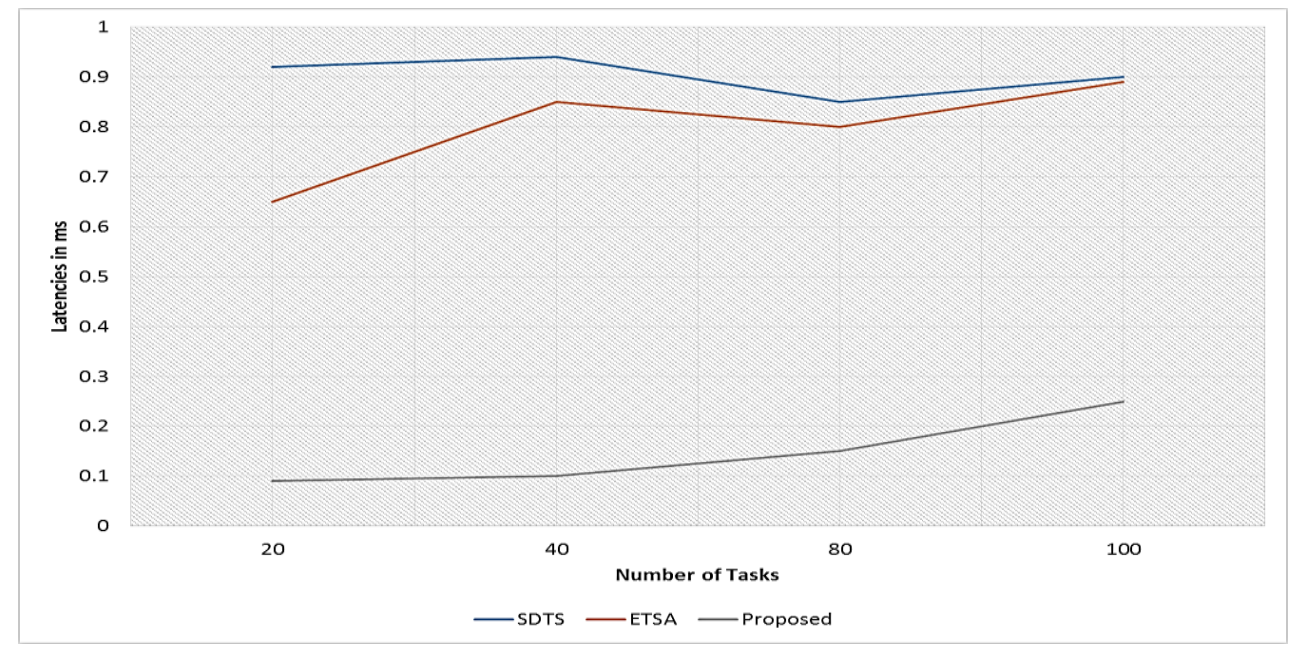

Figure.5 latency Observed 
Journal of trends in Computer Science and Smart technology (TCSST) (2020)

Vol.02/ No. 01

Pages: 26- 36

https://www.irojournals.com/tcsst/

DOI: https://doi.org/10.36548/jtcsst.2020.1.003

The figure. 5 shown above is the latency observed in the proposed method and it is comparison with the existing methods. The latency observed proves that the execution time of the tasks using the proposed method is very much reduced compared to the ETSA and SDTS.

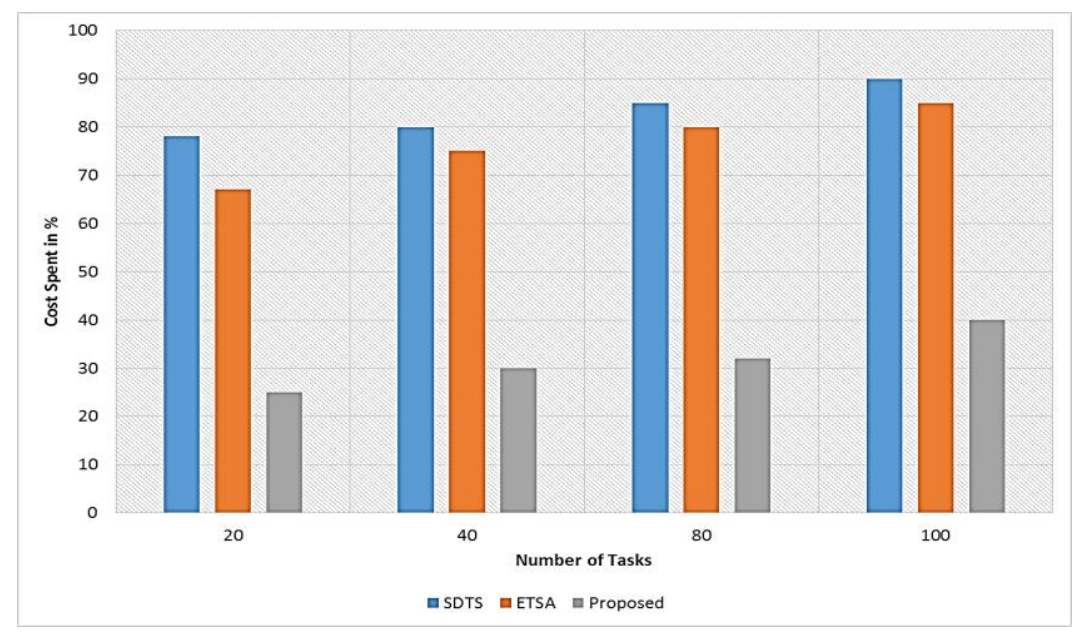

Figure.6 Cost Spent

The figure .6 shows the cost reduction in the tasks offloading achieved by the proposed method compared to the existing ETSA and SDTS.

\section{Conclusion}

To optimize the energy consumption latency incurred and the cost spent to enhance the resources of the mobile devices using the MCC in order to handle computational intensive tasks, the proposed method puts forth the offloading based on double stranded estimation and resource allocation based on the ACO, so as to bring down the energy used in the offloading and satisfy the requirements of the task by executing them in the resource that are compatible with the task respectively, avoiding failure in the execution of the task and completing the tasks before the fixed dead line. The proposed model is evaluated and compared with the existing method on the basis of energy consumption, latency and the cost and was found better. Further

ISSN: 2582-4104 
Journal of trends in Computer Science and Smart technology (TCSST) (2020)

Vol.02/ No. 01

Pages: 26- 36

https://www.irojournals.com/tcsst/

DOI: https://doi.org/10.36548/jtcsst.2020.1.003

the integrating the edge with the cloud to further reduce the latency of more sensitive tasks is devised as the future work of the paper.

\section{References}

[1] Tao, Yaling, Yongbing Zhang, and Yusheng Ji. "Efficient computation offloading strategies for mobile cloud computing." In 2015 IEEE 29th International Conference on Advanced Information Networking and Applications, pp. 626-633. IEEE, 2015.

[2] Hyytiä, Esa, Thrasyvoulos Spyropoulos, and Jörg Ott. "Optimizing offloading strategies in mobile cloud computing." Cryptanalyst (2013).

[3] Smys, S., \& Raj, J. S. (2019). A Stochastic Mobile Data Traffic Model for Vehicular Ad Hoc Networks. Journal of Ubiquitous Computing and Communication Technologies (UCCT), 1(01), 55-63.

[4] Deng, Shuiguang, Longtao Huang, Javid Taheri, and Albert Y. Zomaya. "Computation offloading for service workflow in mobile cloud computing." IEEE transactions on parallel and distributed systems 26, no. 12 (2014): 3317-3329.

[5] Bhalaji, N. (2019). Delay Diminished Efficient Task Scheduling and Allocation for Heterogeneous Cloud Environment. Journal of trends in Computer Science and Smart technology (TCSST), 1(01), 51-62.

[6] Ma, Xiao, Chuang Lin, Xudong Xiang, and Congjie Chen. "Game-theoretic analysis of computation offloading for cloudlet-based mobile cloud computing." In Proceedings of the 18th ACM International Conference on Modeling, Analysis and Simulation of Wireless and Mobile Systems, pp. 271-278. 2015.

[7] Karunakaran, V. "A Stochastic Development of Cloud Computing Based Task Scheduling Algorithm." Journal of Soft Computing Paradigm (JSCP) 1, no. 01 (2019): 41-48.

[8] Barbarossa, Sergio, Paolo Di Lorenzo, and Stefania Sardellitti. "Computation offloading strategies based on energy minimization under computational rate constraints." In 2014 European Conference on Networks and Communications (EuCNC), pp. 1-5. IEEE, 2014.

[9] Barbarossa, Sergio, Stefania Sardellitti, and Paolo Di Lorenzo. "Computation offloading for mobile cloud computing based on wide cross-layer optimization." In 2013 Future Network \& Mobile Summit, pp. 1-10. IEEE, 2013.

[10] Bashar, A. (2019). Secure And Cost Efficient Implementation Of The Mobile Computing Using Offloading Technique. Journal of Information Technology, 1(01), 48-57.

ISSN: 2582-4104 
Journal of trends in Computer Science and Smart technology (TCSST) (2020)

Vol.02/ No. 01

Pages: 26- 36

https://www.irojournals.com/tcsst/

DOI: https://doi.org/10.36548/jtcsst.2020.1.003

[11] Gao, Bo, Ligang He, Limin Liu, Kenli Li, and Stephen A. Jarvis. "From mobiles to clouds: Developing energy-aware offloading strategies for workflows." In 2012 ACM/IEEE 13th International Conference on Grid Computing, pp. 139-146. IEEE, 2012.

[12] Khan, Minhaj Ahmad. "A survey of computation offloading strategies for performance improvement of applications running on mobile devices." Journal of Network and Computer Applications 56 (2015): 28-40.

[13] Kamoun, Mohamed, Wael Labidi, and Mireille Sarkiss. "Joint resource allocation and offloading strategies in cloud enabled cellular networks." In 2015 IEEE International Conference on Communications (ICC), pp. 5529-5534. IEEE, 2015.

[14] Ge, Yang, Yukan Zhang, Qinru Qiu, and Yung-Hsiang Lu. "A game theoretic resource allocation for overall energy minimization in mobile cloud computing system." In Proceedings of the 2012 ACM/IEEE international symposium on Low power electronics and design, pp. 279-284. 2012.

[15] Shi, Yan, Shanzhi Chen, and Xiang Xu. "MAGA: A mobility-aware computation offloading decision for distributed mobile cloud computing." IEEE Internet of Things Journal 5, no. 1 (2017): 164-174.

[16] Wu, Huaming, William Knottenbelt, and Katinka Wolter. "Analysis of the energy-response time tradeoff for mobile cloud offloading using combined metrics." In 2015 27th International Teletraffic Congress, pp. 134-142. IEEE, 2015.

[17] Xia, Siyu, Mingyuan Jiu, and Chenglong Fan. "Single and Double-Threaded Offloading Strategies and Computational Analysis in Mobile Cloud Computing." In Journal of Physics: Conference Series, vol. 1437, no. 1, p. 012018. IOP Publishing, 2020.

[18] Feng, Jingyun, Zhi Liu, Celimuge Wu, and Yusheng Ji. "AVE: Autonomous vehicular edge computing framework with ACO-based scheduling." IEEE Transactions on Vehicular Technology 66, no. 12 (2017): 10660-10675.

ISSN: 2582-4104 